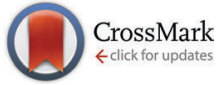

Cite this: Phys. Chem. Chem. Phys., 2014, 16, 25342

Received 16th September 2014 Accepted 16th October 2014

DOI: $10.1039 / c 4 c p 04156 a$

www.rsc.org/pccp

\title{
Highly efficient green-light ionization of an aryl radical anion: key step in a catalytic cycle of electron formation $\dagger$
}

\author{
Christoph Kerzig and Martin Goez*
}

\begin{abstract}
A sustainable generation of hydrated electrons with green light would allow solar-driven applications of this potent reductant, such as the detoxification of halogenated organic waste. Using two-color laser flash photolysis, we have studied the photoionizations of the 1,5-naphthalene disulfonate radical anion and triplet with $532 \mathrm{~nm}$ as well as $355 \mathrm{~nm}$. The radical anion is prepared by reducing the triplet with the bioavailable ascorbate monoanion under physiological conditions; its photoionization recovers the starting substrate, so turns the reaction sequence into a catalytic cycle. A comparison of the four ionizations suggests that their efficiency is strongly influenced by the electronic configuration of the state ejecting the electron. The quantum yield for ionizing the radical anion with $532 \mathrm{~nm}(0.27)$ is at least four times higher than for the very few known examples of such green-light ionizations and comparable to the most efficient UV ionizations known to date, so this system might represent a breakthrough towards the "green" production of hydrated electrons.
\end{abstract}

\section{Introduction}

Aqueous photoionizations hold great promise because they permit a controlled release under natural or even physiological conditions of the hydrated electron $\mathbf{e}_{\mathrm{aq}}{ }^{{ }^{-}}$, a species that is as strong and reactive a reductant as activated alkali metals and has a natural life long enough for bimolecular attacks on additives. ${ }^{1}$ These unique properties of $\mathbf{e}_{\mathbf{a q}}{ }^{\boldsymbol{}}{ }^{-}$have already been put to use under laboratory conditions for the efficient degradation of chlorinated ${ }^{2,3}$ and even fluorinated ${ }^{4}$ organics, which characteristically are toxic, yet extremely persistent, so accumulate in the environment. Hence, the optimization of photoionizations with respect to the quantum yields and operating wavelengths could eventually result in applications of $\mathbf{e}_{\mathbf{a q}} \mathbf{e}^{--}$on a larger scale, for instance, for the reductive detoxification of organic pollutants.

With that ultimate aim in mind, solar-driven photoionizations appear most desirable. Thermodynamically, however, electron detachment from a stable compound by a single photon demands wavelengths in the UV region, where the intensity of the terrestrial solar spectrum sharply drops. In direct consequence of that energetic aspect, a highly excited state of the substrate is involved,

Martin-Luther-Universität Halle-Wittenberg, Institut für Chemie, Kurt-Mothes-Str. 2, D-06120 Halle (Saale), Germany. E-mail: martin.goez@chemie.uni-halle.de

$\dagger$ Electronic supplementary information (ESI) available: Experimental details, absorption spectra and properties of the relevant chemical species. See DOI: $10.1039 / \mathrm{c} 4 \mathrm{cp} 04156 \mathrm{a}$ and as long as that state is still a bound or resonance state ${ }^{5}$ electron ejection thus has to compete with extremely fast radiationless deactivation. That argument can rationalize why even at $266 \mathrm{~nm}$, which supplies ample photon energy but is completely absorbed by the ozone layer, the quantum yields of reported monophotonic ionizations never exceed $30 \%$ and are often more than an order of magnitude lower..$^{6-9}$

Simultaneously meeting the energetic requirement and avoiding a highly excited state with its associated short life seems only possible by using two photons of lower energy in succession and storing the energy of the first photon in an intermediate that is not an excited state. This concept is substantiated by our repeated observation that radical anions of ketones and quinones exhibit five- to ten-fold higher photoionization quantum yields than the corresponding triplets. ${ }^{10-14}$ A further advantage of these chemical intermediates is the absence of photophysical deactivation, tantamount to an intrinsically longer life, which maximizes the probability of absorbing the ionizing photon even at low photon fluxes. What finally singles out radical anions as special is the fact that their ionization regenerates their parent compounds, which turns the whole reaction sequence, absorption - reductive quenching by a sacrificial donor - absorption, into a catalytic cycle, so in principle allows the production of unlimited amounts of $\mathbf{e}_{\mathbf{a q}}{ }^{\bullet-}$ without consumption of the photoactive substrate.

The following considerations apply to the optimization of such a cycle: because the quantum yield of excitation is always unity, the first photon may lie in the UV range, but the color of 
the ionizing photon should be optimally matched to the solar spectrum, ${ }^{15}$ that is, should ideally be green. The latter in turn calls for an energy-rich radical anion, as the energy of a green photon (2.33 eV at $532 \mathrm{~nm}$ ) falls slightly short of the formation energy of $\mathbf{e}_{\mathbf{a q}}{ }^{\bullet-}\left(2.77 \mathrm{~V} v s\right.$. NHE). ${ }^{1}$ To maximize the radical anion yield, the excited substrate should undergo efficient intersystem crossing to the triplet state, because that state can be quenched with much lower donor concentrations owing to its longer life and produces a triplet radical-ion pair, for which reverse electron transfer is spin-forbidden. ${ }^{16}$ The donor, being the only species consumed in the cycle, should be inexpensive and preferentially bioavailable; ideally, it should quench under physiological conditions. Lastly, the ionization step itself should be monophotonic because otherwise two photons would have to impinge on the radical anion within nanoseconds, which can only be achieved by pulsed laser illumination.

All these criteria are fulfilled by the system that we have investigated in this work, using two-color laser flash photolysis. The substrate chosen is the water-soluble 1,5-naphthalene disulfonate $\left(\mathbf{N D S}^{\mathbf{2}}\right)$, which efficiently forms a high-energy triplet state ${ }^{3} \mathbf{N D S}^{2-}$ (quantum yield, 0.88; triplet energy, $2.58 \mathrm{eV}$ ) ${ }^{17}$ upon excitation with $308 \mathrm{~nm}$. As we have found, the triplet is quenched by the ascorbate monoanion $\left(\mathbf{H A s c}^{-}\right)$at neutral $\mathrm{pH}$ to give the radical anion $\mathbf{N D S}^{\mathbf{3}^{3}-}$. Because that aryl radical anion is more energy-rich by at least $60 \mathrm{~kJ} \mathrm{~mol}^{-1}$ than the carbonyl radical anions reported on earlier by us, ${ }^{10,11,18}$ which required near-UV light for their ionization, it appears as an ideal candidate for an ionization with green light.

\section{Results and discussion}

\subsection{Reaction mechanism}

Fig. 1a displays the concentration traces of the reaction products $\mathbf{N D S}^{\bullet{ }^{-}-}$and $\mathbf{e}_{\mathbf{a q}}{ }^{\bullet-}$ in a representative two-color laser flash photolysis experiment on our substrate $\mathbf{N D S}^{\mathbf{2}-}$ in the presence of the quencher HAsc $^{-}$. The protocol for obtaining these concentrations is described in Section S2.5 of the ESI. $\dagger$

The function of the first laser pulse $(308 \mathrm{~nm})$ is to convert the substrate into its triplet state ${ }^{3} \mathbf{N D S}^{\mathbf{2}}$; at the pulse intensity selected by us that conversion is maximized (15\%) while the undesired UV ionization of the excited naphthalene ${ }^{19}$ during that pulse is minimized $(0.3 \%)$. The ensuing interpulse delay serves to accumulate the radical anion $\mathbf{N D S}^{\mathbf{0}^{-}-}$through the quenching of ${ }^{3} \mathbf{N D S}^{\mathbf{2}-}$ by $\mathbf{H A s c}^{-}$; we chose that waiting period and the quencher concentration for optimum concentrations of NDS $^{\mathbf{0}^{-}}$at the moments the two lasers fire (as high as possible at the start of the second pulse, to enhance the sensitivity; as low as possible during the first pulse, to suppress a photoionization of the radical anion already by that pulse). The second laser pulse $(532 \mathrm{~nm})$ finally brings about a green-light ionization of the radical anion on a large scale, as is evidenced in Fig. 1a by the bleaching of more than $50 \%$ of $\mathbf{N D S}^{\mathbf{e}^{3-}}$ accompanied by the formation of the same amount of $\mathbf{e}_{\mathbf{a q}}{ }^{\bullet-}$.

No electrons are produced in control experiments without radical anions, that is, when the quencher or the first laser pulse are absent. The radical anions are also the only species converted by the second flash: besides them, only the triplet would absorb at $532 \mathrm{~nm}$ (for all spectra, see Section S2 of the ESI $\dagger$ ) but features a significantly lower molar absorption coefficient and has largely vanished at the end of the interpulse delay. All these findings are in perfect accordance with the catalytic cycle of photoionization of Fig. $1 \mathrm{~b}$.

\subsection{Radical anion formation and decay}

As has emerged, the photoionizable radical anion $\mathbf{N D S}^{\mathbf{*}}{ }^{-}$is the key species in the catalytic cycle of Fig. 1b. To prepare $\mathbf{N D S}^{\bullet{ }^{3}-}$, we used reductive quenching of the triplet ${ }^{3} \mathbf{N D S}^{2-}$ by the ascorbate monoanion HAsc $^{-}$(eqn (1))

$$
{ }^{3} \mathrm{NDS}^{2-}+\mathrm{HAsc}^{-} \stackrel{k_{\mathrm{q}}}{\longrightarrow} \mathrm{NDS}^{\bullet 3^{-}}+\mathrm{HAsc}^{\bullet}
$$
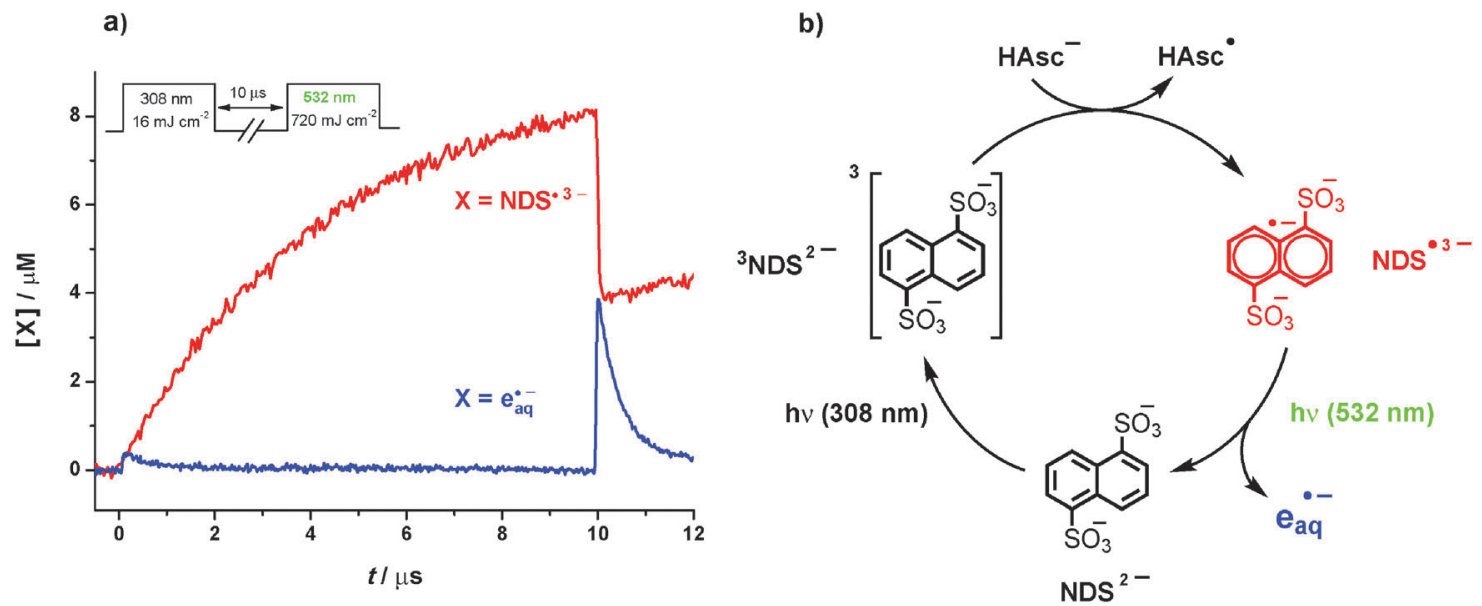

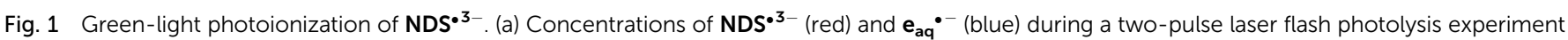
on a deoxygenated aqueous solution of $1.4 \times 10^{-4} \mathrm{M} \mathrm{NDS}^{2-}$ and $2 \mathrm{mM} \mathrm{HAsc}{ }^{-}$at $\mathrm{pH} 7.6$ with the pulse scheme shown as the inset. The small amount of $\mathbf{e}_{\mathbf{a q}}{ }^{--}$produced by the first flash is due to a side reaction (UV photoionization of the excited substrate); the $\mathbf{e}_{\mathrm{aq}}{ }^{{ }^{-}-}$yield through green-light ionization of the radical anion by the second flash is higher by one order of magnitude. (b) Cyclic photoionization mechanism for NDS ${ }^{2-}$ in the presence of HAsc acting as electron donor. For further details, see text and ESI. $\dagger$ 
Our attempts to follow that reaction by monitoring the concentrations of its products were obstructed by the subsequent protonation of $\mathbf{N D S}^{\bullet-}$, which removes its absorptions in the visible region and changes the UV absorptions in the range suitable for observing $\mathbf{H A s c}^{\bullet}$. That protonation is mechanistically complex, fast enough to modify the buildup of $\mathbf{N D S}^{\bullet \mathbf{}} \mathbf{3}^{-}$ in Fig. 1a, and not suppressible by a faster quenching and a shorter interpulse delay because we already used the maximum concentration of HAsc $^{-}$compatible with homogeneous absorption of the initial $308 \mathrm{~nm}$ laser pulse.

In contrast, the triplet provides a reliable observable for investigating the quenching. At the maximum of its visible band, $440 \mathrm{~nm}$, the only other absorbing species is $\mathbf{N D S}^{\mathbf{0 3}^{-}}$, and the latter in turn has a band centered at $725 \mathrm{~nm}$, where all other species are transparent (compare, the spectra in Section S2 of the ESI $\dagger$ ). Hence, the triplet decay is accessible from traces recorded at $440 \mathrm{~nm}$ and $725 \mathrm{~nm}$ with all other experimental parameters unchanged: the contribution of $\mathbf{N D S}^{\mathbf{*}-}$ to the $440 \mathrm{~nm}$ trace is obtained by multiplying the $725 \mathrm{~nm}$ trace with the ratio of molar absorption coefficients $\left(\varepsilon_{440} / \varepsilon_{725}=1.36\right)$, and subtracting it from the $440 \mathrm{~nm}$ trace extracts the pure triplet signal with optimum sensitivity and regardless of how the concentration of $\mathbf{N D S}^{\mathbf{}^{3-}}$ is affected by its protonation. Fig. 2a illustrates that separation, which we found to isolate a firstorder decay in accordance with eqn (1).

To determine the quenching rate constant $k_{\mathrm{q}}$, we repeated that procedure with different quencher concentrations and analyzed the concentration dependence of the triplet lifetime (Fig. 2b, main plot and inset). The Stern-Volmer plot is linear, indicating purely dynamic quenching, with a Stern-Volmer constant of $2.70 \times 10^{3} \mathrm{M}^{-1}$; with the unquenched lifetime of $57.5 \mu$ s under our experimental conditions, a quenching rate constant of $4.7 \times 10^{7} \mathrm{M}^{-1} \mathrm{~s}^{-1}$ thus results.

The reduction of ${ }^{3} \mathbf{N D S}^{2-}$ by $\mathbf{H A s c}^{-}$is rather slow, considering that photoinduced electron transfer reactions are frequently diffusion-controlled. An explanation is provided by its Gibbs energy $\Delta G^{\circ},{ }^{20}$ in $\mathrm{eV}$ (eqn (2)),

$$
\Delta G^{\circ}=E^{\circ}\left(\mathbf{H A s c}^{\bullet} / \mathbf{H A s c}^{-}\right)-E^{\circ}\left(\mathbf{N D S}^{2-} / \mathbf{N D S}^{\bullet-}\right)-\Delta E_{0,0}+w_{\mathrm{P}}-w_{\mathrm{R}}
$$

With the standard potentials $E^{\circ}$ for reduction of the donor radical and the acceptor $\left(+0.30 \mathrm{~V}^{21}\right.$ and $-1.97 \mathrm{~V},{ }^{22}$ both $v s$. NHE $)$ and the triplet energy $\left(E_{0,0}=2.58 \mathrm{eV}\right),{ }^{17,23}$ the distance-independent base value of $\Delta G^{\circ}$ is calculated to be $-0.31 \mathrm{eV}$, to which the reactant Coulombic term $-w_{\mathrm{R}}$ has to be added (the counterpart for the products, $w_{\mathrm{P}}$, is zero because HAsc $^{\bullet}$ is uncharged). As $w_{\mathrm{R}}$ amounts to $(0.36 \mathrm{eV}) / a$ when the reaction distance $a$ is specified in ångström units, a negligible contribution of about $-0.05 \mathrm{eV}$ is expected using a typical distance ${ }^{16}$ of $7 \AA$. The slow rate should thus reflect the low exergonity of the reaction. Consistent with the involvement of a highly charged activated complex there is a remarkable acceleration of the quenching with increasing ionic strength of the solution (kinetic salt effect): ${ }^{24}$ upon addition of only $75 \mathrm{mM}$ of sodium chloride, we observed a doubling of $k_{\mathrm{q}}$.

Despite the moderate value of $k_{\mathrm{q}}$, the triplet is still quenched efficiently by fairly low donor concentrations because its natural life is so long. The half-quenching concentration of HAsc ${ }^{-}$is as small as $370 \mu \mathrm{M}$, and the degree of quenching rises to $85 \%$ under the conditions of Fig. 1a. However, a significant fraction of quenching events does not yield free radical anions of our substrate. As Fig. 3a shows, the concentration trace for $\mathbf{N D S}^{\bullet \mathbf{}}$ is describable by a formation with the same rate as the triplet decay but an efficiency $\eta$ of only 0.56 , and by a subsequent slow decrease with a half life of $25 \mu \mathrm{s}$.

The unexpectedly low $\eta$ might be caused by two different reactions of the spin-correlated radical pair $\overline{\mathbf{N D S}^{\bullet 3-} \mathbf{H A s c}^{\bullet}}$ (eqn (3)), which is the initial product of electron-transfer quenching of the triplet. The pair is born with the multiplicity of its precursors, so in a triplet state. Escape from the cage a)

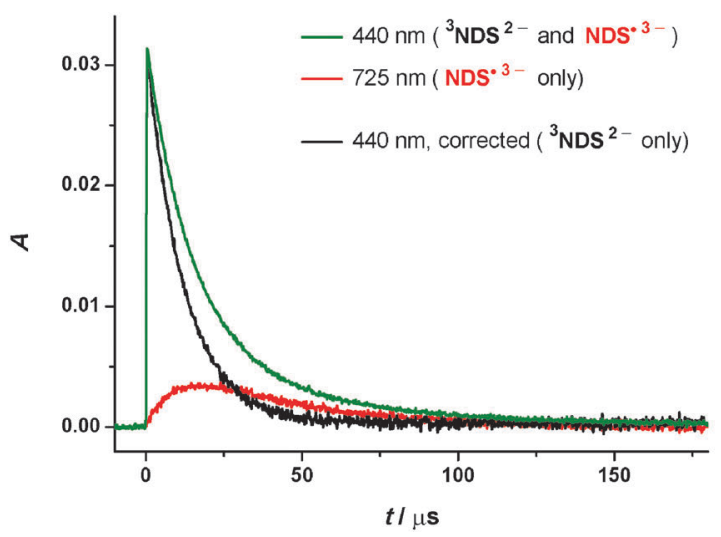

b)

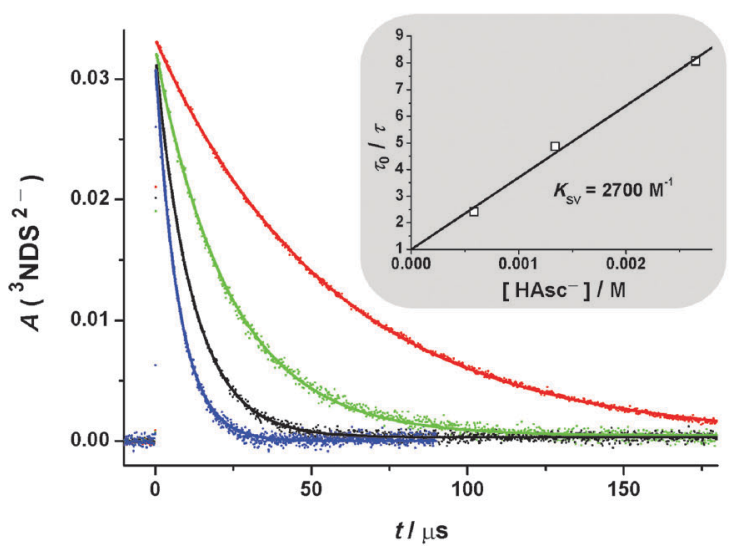

Fig. 2 (a) Extracting the pure triplet signal at $440 \mathrm{~nm}$ (black) from experimental absorption traces at $440 \mathrm{~nm}$ (green) and $725 \mathrm{~nm}$ (red) by weighing the latter trace with the factor 1.36 and subtracting the result from the former trace. Experimental conditions, laser flash photolysis (308 $\mathrm{nm}, 4.5 \mathrm{~mJ} \mathrm{~cm}^{-2}$ ) of an $\mathrm{N}_{2} \mathrm{O}$-saturated solution of $1.4 \times 10^{-4} \mathrm{M} \mathrm{NDS}^{2-}$ quenched with $1.34 \mathrm{mM} \mathrm{HAsc}^{-}$. (b) Influence of the concentration of $\mathrm{HAsc}^{-}$on the lifetime $\tau$ of ${ }^{3} \mathrm{NDS}^{2-}$. Main plot, ${ }^{3} \mathrm{NDS}^{2-}$ decays without (red) and with $\mathrm{HAsc}^{-}$(light green, $0.59 \mathrm{mM}$; black, $1.34 \mathrm{mM}$; blue, $2.65 \mathrm{mM}$ ) obtained by the method shown in subfigure $a$, with all other parameters the same as there; dots, experimental data; solid lines, best-fit functions given by $A_{0} \times \exp [-t / \tau]$. Inset, Stern-Volmer plot based on the lifetimes $\tau$ determined in the main plot, with the unquenched lifetime $\tau_{0}$. For further details, see text. 
a)

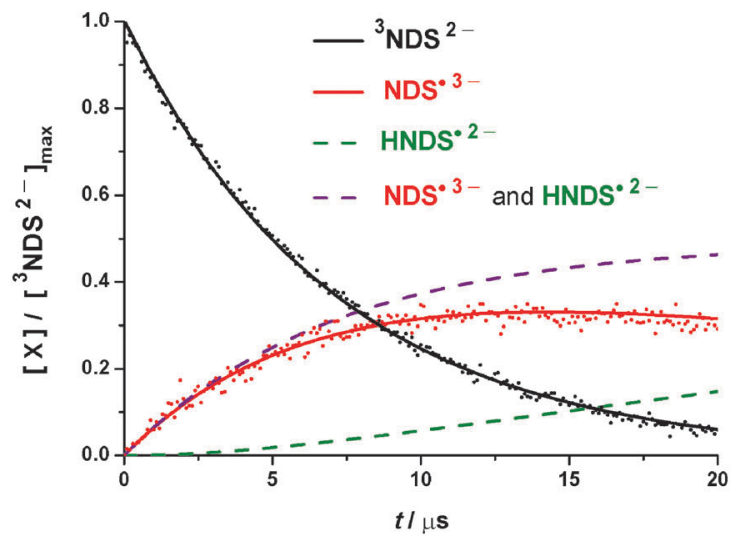

b)

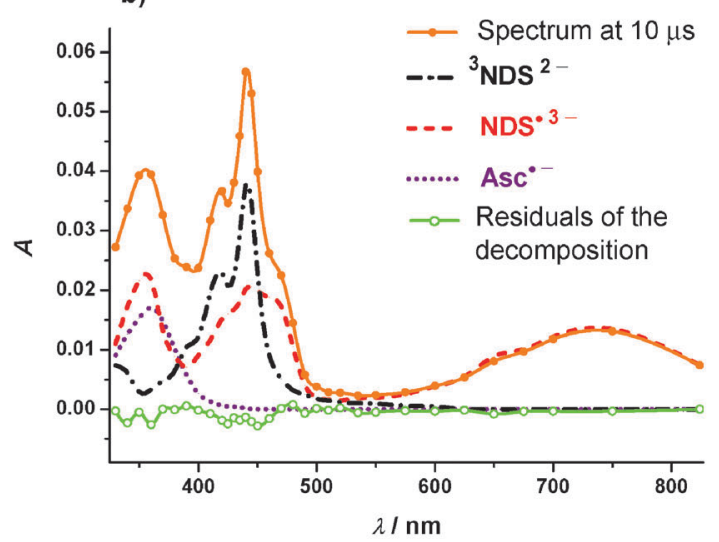

Fig. 3 Radical anion formation and decay in the quenching of ${ }^{3} \mathrm{NDS}^{2-}$ by $\mathrm{HAsc}^{-}$under the conditions of Fig. 1a but with $2.65 \mathrm{mM}$ of $\mathrm{HAsc}^{-}$. (a) Measured and simulated concentration traces for species $X$, normalized to the initial triplet concentration. Black and red dots, experimental data points for the triplet and the radical anion; black solid line, fit of $\exp \left[-k_{1} t\right]$ to the triplet decay, best-fit parameter $k_{1}=1.42 \times 10^{5} \mathrm{~s}^{-1}$; red solid line, fit of $R(t)$, $R(t)=\eta \frac{k_{1}}{k_{1}-k_{2}}\left(\exp \left[-k_{2} t\right]-\exp \left[-k_{1} t\right]\right)$ to the radical-anion curve with $k_{1}$ fixed at the previous value, best-fit parameters $\eta=0.56$ and $k_{2}=2.8 \times 10^{4} \mathrm{~s}^{-1}$; purple dashed line, calculated concentration $S(t)$ of unprotonated plus protonated radical anion, i.e., $S(t)=\eta\left(1-\exp \left[-k_{1} t\right]\right)$; dark green dashed line, calculated concentration trace for the protonated radical anion, i.e., difference between the two preceding curves. At $10 \mu$ s, the concentration ratio $S(t) / R(t)$, i.e., of the substrate-derived radicals in all their forms and in the form of the radical anion only, is 1.2 with the best-fit parameters given above. (b) Kinetic spectrum at $10 \mu \mathrm{s}$ after the $308 \mathrm{~nm}$ pulse (orange solid line and filled dots); contributions of ${ }^{3} \mathrm{NDS}^{2-}$ (black dash-dotted line), NDS ${ }^{\cdot 3-}$ (red dashed line), and $\mathbf{A s c}^{\bullet-}$ (purple dotted line); residuals of the decomposition (light green solid line and open dots). Further explanation, see text.

affords the desired free radicals $\mathbf{N D S}^{\mathbf{0}^{-3}}$ but has to compete with two geminate processes: reverse electron transfer leads back to the starting materials but is only possible after intersystem crossing, whereas in-cage proton transfer transforms the primary pair into a secondary one that is no longer capable of releasing $\mathbf{N D S}^{\mathbf{\bullet}^{3-}}$; in ketone-amine systems we have already studied such transformations, ${ }^{25}$ which are not subject to any spin restrictions and occur within the spin-correlated life of the pairs, that is, on a subnanosecond timescale.

$$
\mathbf{N D S}^{2-}+\text { HAsc }^{-} \underset{\text { transfer }}{\stackrel{\text { electron isc }}{\longleftarrow}} \frac{3}{\text { NDS }^{\bullet-3-} \mathbf{H A s c}^{\bullet}} \underset{\text { transfer }}{\stackrel{\text { proton }}{\longrightarrow}} \frac{3}{\mathbf{H N D S}^{\bullet-2} \mathbf{A s c}^{\bullet-}}
$$

The decomposition into components of the transient spectrum displayed in Fig. $3 \mathrm{~b}$ rules out the proton-transfer pathway and sustains the electron-transfer route. First, a sharp peak centered at about $340 \mathrm{~nm}$, which has been observed for the protonated radical anions of naphthalene and naphthalene2-sulfonate, ${ }^{26,27}$ would be expected for HNDS $^{\bullet 2-}$ but is not yet noticeable at the post-flash delay used in Fig. 3b; because the in-cage proton transfer would have to yield that transient in approximately the same amount as $\mathbf{N D S}^{\mathbf{0}^{-3}}$ to be consistent with the observed $\eta$, that signal should have been quite prominent. Second, the $1: 1$ concentration ratio between $\mathbf{A s c}^{\bullet-}$ (which is the only observable form of the free ascorbate-derived radicals; see Section S2.4 of the ESI $\dagger$ ) and NDS $^{\mathbf{0}^{-3}}$ imposed by the primary quenching remains unchanged by back electron transfer, whereas in-cage proton transfer as well as protonation of free $\mathbf{N D S}^{\mathbf{*}^{3-}}$ increase that ratio by removing $\mathbf{N D S}^{\boldsymbol{\bullet}^{3-}}$ without influencing the concentration of $\mathbf{A s c}^{\bullet-}$. Under the experimental conditions of Fig. $3 \mathrm{~b}$, the protonation of free $\mathbf{N D S}^{\mathbf{0}^{3-}}$ alone

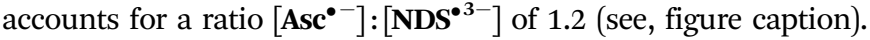
That value would not alter if the observed $\eta$ were due to electron return, but would have to rise to 2.1 if in-cage proton transfer were responsible. By decomposing the spectrum as shown in Fig. 3b, we determined a ratio of 1.2 , which unambiguously identifies the back electron transfer as the reason for the low $\eta$.

As a corollary, intersystem crossing of $\overline{\mathbf{N D S}^{\bullet 3-} \mathbf{H A s c}^{\bullet}}$ by the radical-pair mechanism ${ }^{28}$ must be unusually fast, which might reflect a substantial increase of the $g$ value by the sulfonation. On the other hand, in view of the thermodynamic instability of aryl radical anions in water (the $\mathrm{p} K_{\mathrm{a}}$ values of the corresponding. acids are significantly larger than 14$),{ }^{29,30}$ the protonation of $\mathbf{N D S}^{\mathbf{0}^{3-}}$ is surprisingly slow. Both processes are certainly undesirable because they reduce the overall quantum yield but they do not necessarily compromise the stability of the system: the former quantitatively regenerates the substrate, while the latter is slow enough to be rendered unimportant at our light intensities, where $\mathbf{N D S}^{{ }^{\mathbf{3}-}}$ is photoionized much faster than it can be protonated.

\subsection{Green-light photoionization}

To check whether the photoionization of $\mathbf{N D S}^{\boldsymbol{0}^{3-}}$ is accompanied by side reactions, we recorded kinetic spectra as in Fig. $3 \mathrm{~b}$ also after the second laser pulse, which gave no indication whatsoever for the presence of any other species besides those included in the mechanism of Fig. 1b. We take this as evidence that ionization is the only chemical reaction pathway of excited $\mathbf{N D S}^{\mathbf{*}}{ }^{\mathbf{}}$. Based on these findings, we determined the molar absorption coefficient of $\mathbf{N D S}^{\mathbf{0}^{3-}}$ relative to that of $\mathbf{e}_{\mathrm{aq}}{ }^{-\boldsymbol{}}$, that is, by comparing bleaching and electron formation (see, Section S2.3 of the ESI $\dagger$ ). That calibration eliminates all errors due to uncertainties of the optical path length and imperfections of 

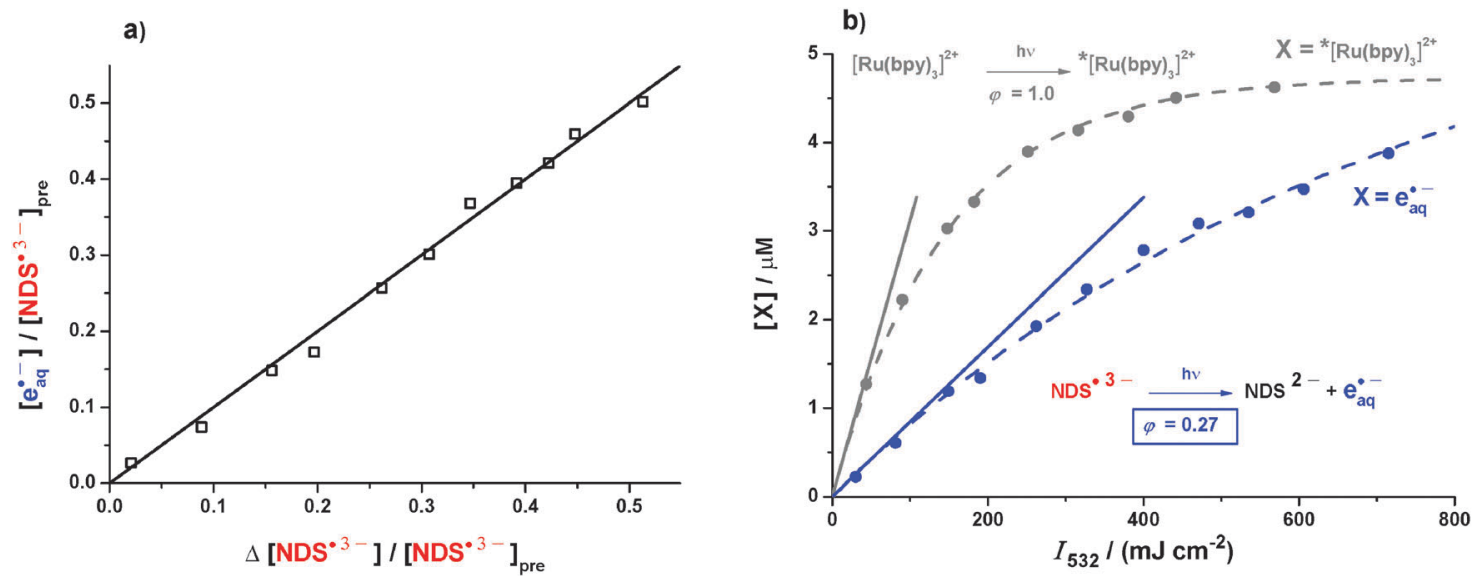

Fig. 4 Intensity dependent measurements of the green-light ionization of $\mathbf{N D S}^{\boldsymbol{\bullet}^{3-}}$. (a) $\mathbf{e}_{\mathrm{aq}}{ }^{\boldsymbol{\bullet}^{-}}$formation as a function of $\mathbf{N D S}^{\mathbf{0}^{3-}}$ bleaching at different intensities of the ionizing laser pulse; other experimental parameters as in Fig. 1a. [NDS $\left.{ }^{\cdot 3-}\right]_{\text {pre }}$ is the prepulse concentration of the radical anion. (b) Relative actinometry with $\left[\mathrm{Ru}(\mathrm{bpy})_{3}\right]^{2+}$ as standard. Filled circles, intensity dependences of $\mathbf{e}_{\mathrm{aq}}{ }^{-{ }^{-}}$formation from $\mathrm{NDS}^{{ }^{* 3-}}$ (blue) and MLCT formation from $\left[\mathrm{Ru}(\mathrm{bpy})_{3}\right]^{2+}$ (gray). Initial absorbance of both solutions at $532 \mathrm{~nm}, 4.0 \times 10^{-3} \mathrm{~cm}^{-1}$. The dashed lines are the best-fit functions $[X]_{\max }\left(1-\exp \left[-a I_{532}\right]\right)$ with the maximum concentration $[X]_{\max }$ and the fit parameter $a$. Solid lines, initial slopes $a[X]_{\max }$, with the photoionization quantum yield given by their ratio, 0.27 . Further details, see text.

the excitation homogeneity. With the molar absorption coefficient so obtained the equality of one radical anion being consumed per one electron produced holds at any laser intensity, as the mechanism of Fig. 1b demands; this is established by the plot of Fig. 4a.

The dependence of the electron yield on the intensity of the green laser flash can be seen in Fig. 4b. It evidently features a linear low-intensity regime with a straight-line extrapolation to the origin, ${ }^{31}$ so identifies the green-light ionization of the radical anion as monophotonic. This is also very plausible because for that reaction, eqn (4), the sum of the photon energy $(2.33 \mathrm{eV}$ at $532 \mathrm{~nm})$ and the energy gain by the recovery of the substrate from the radical anion $(1.97 \mathrm{eV})^{22}$ greatly surpasses the formation energy of $\mathbf{e}_{\mathrm{aq}}{ }^{--}(2.77 \mathrm{eV}){ }^{1}$

$$
\mathbf{N D S}^{\bullet-3} \stackrel{532 \mathrm{~nm}}{\longrightarrow} \mathbf{N D S}^{2-}+\mathbf{e}_{\mathrm{aq}}{ }^{--}+1.53 \mathrm{eV}
$$

The same considerations help rationalize why the triplet is not ionized with green light according to eqn (5) despite a weak absorption at $532 \mathrm{~nm}$ : triplet energy $(2.58 \mathrm{eV})^{17}$ plus photon energy barely outweigh the energetic requirements for the oxidation of the substrate $\left(E^{\circ}\left(\mathbf{N D S}^{\bullet-} / \mathbf{N D S}^{2-}\right)=2.03 \mathrm{eV} v s \text {. NHE }\right)^{32}$ and the formation of $\mathbf{e}_{\mathbf{a q}}{ }^{\cdot-}$,

$$
{ }^{3} \mathbf{N D S}^{2-} \stackrel{532 \mathrm{~nm}}{\longrightarrow} \mathbf{N D S}^{\bullet-}+\mathbf{e}_{\mathbf{a q}^{--}}+0.11 \mathrm{eV}
$$

We measured the photoionization quantum yield of $\mathbf{N D S}^{\mathbf{}^{3-}}$ by relative actinometry with ruthenium(tris)bipyridine as the reference. Upon green-light excitation, $\left[\mathrm{Ru}(\mathrm{bpy})_{3}\right]^{2+}$ quantitatively produces a luminescent metal-to-ligand charge-transfer excited state MLCT, ${ }^{33}$ which does not decompose even at the highest laser intensities accessible with our setup; ${ }^{34}$ obtaining the MLCT concentration from the luminescence is straightforward because the system is easily driven so near to complete conversion to MLCT that a reliable calibration curve can be recorded. To this end, Fig. $4 \mathrm{~b}$ also includes the results of variable-intensity laser flash photolysis on a solution of $\left[\mathrm{Ru}(\mathrm{bpy})_{3}\right]^{2+}$ at the same optical density at $532 \mathrm{~nm}$ as $\mathbf{N D S}^{\bullet{ }^{\circ-}}$.

The ratio of initial slopes of the two intensity dependences, for $\mathbf{e}_{\mathrm{aq}}{ }^{{ }^{-}}$formation from $\mathbf{N D S}^{\boldsymbol{\bullet}^{3-}}$ and for MLCT formation from $\left[\mathrm{Ru}(\mathrm{bpy})_{3}\right]^{2+}$, directly gives the quantum yield for green-light ionization of $\mathbf{N D S}^{\mathbf{*}-}$; saturation effects are absent in this lowintensity range, and as with the above determination of the molar absorption coefficient of $\mathbf{N D S}^{\mathbf{*}-}$, the comparison method cancels excitation imperfections. The obtained quantum yield $\varphi_{532}$ of 0.27 outperforms the highest ever reported for a monophotonic ionization in the green (the doubly deprotonated terephthalate radical anion, 0.07$)^{35}$ by as much as a factor of four.

\subsection{UV photoionization}

By using $355 \mathrm{~nm}$ instead of $532 \mathrm{~nm}$ we add $1.17 \mathrm{eV}$ to the photon energy, making the photoionization of the triplet in the UV nearly as exergonic as that of the radical anion in the green (1.28 eV vs. $1.53 \mathrm{eV}$, compare eqn (4) and (5)), and likewise raising the exergonicity of the radical anion photoionization to as much as $2.70 \mathrm{eV}$. Yet, the absence of thermodynamic restrictions does not remove the disparity of the ionization efficiencies, as is evidenced by Fig. 5 .

Fig. 5a compares kinetic traces recorded during $355 \mathrm{~nm}$ photoionization of the triplet and of the radical anion, where we applied the photoionizing pulses with intensities compensating for the different absorptions of ${ }^{3} \mathbf{N D S}^{2-}$ and $\mathbf{N D S}^{{ }^{* 3-}}$. Although the same absolute amount of each intermediate is thus excited, the bleaching is seen to be about five times larger in the case of the radical anion. To ensure that this effect is not caused by the formation of an absorbing product in the triplet photoionization, we performed that experiment in strongly basic solution (at $\mathrm{pH}$ 12.3), where the formal radical cation NDS $^{\boldsymbol{0}^{-}}$of the substrate is rapidly scavenged by hydroxide ions to give a species that is transparent in the visible range. ${ }^{19}$ 
a)

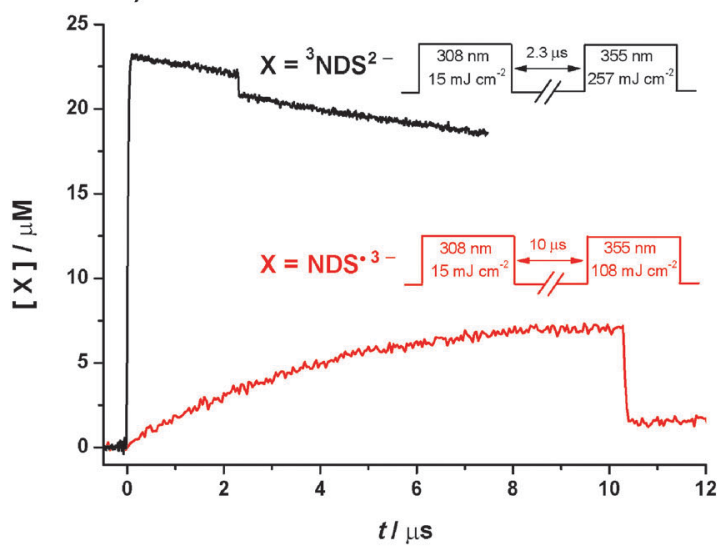

b)

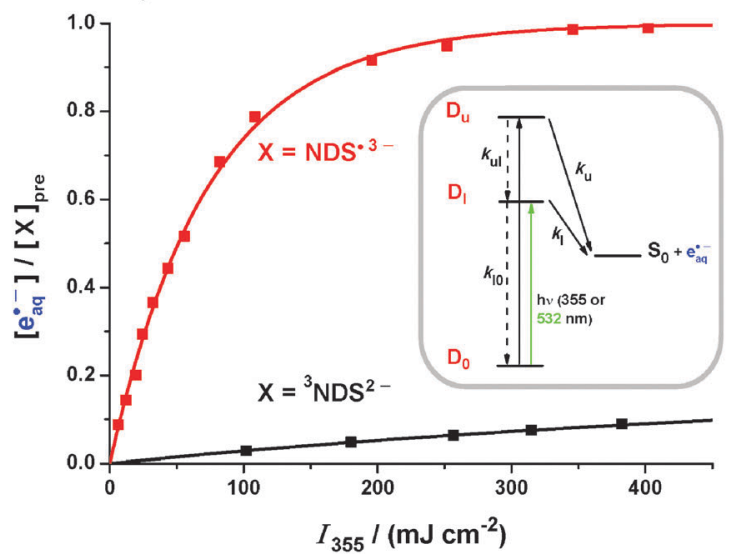

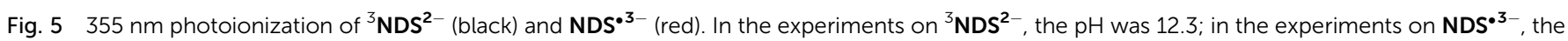
donor concentration was $2.1 \mathrm{mM}$; all other parameters were as in Fig. 1a except for the pulse sequences given in subfigure 5a. (a) Experimental concentration traces and pulse sequences. (b) Main plot, electron concentrations normalized to the prepulse concentration of the respective electron precursor $X$ as functions of the intensity $I_{355}$ of the ionizing pulse. Inset, kinetic scheme for radical-anion photoionization through two adjacent autoionizing states $D_{u}$ and $D_{1}$. Further explanation, see text.

Kinetic spectra recorded before and after the bleaching were identical, and their decay characteristics were also unchanged. Further control experiments showed that the donor-derived radical anion $\mathbf{A s c}^{\mathbf{C}^{-}}$is inert at $355 \mathrm{~nm}$. Hence, the observed bleaching difference reflects a difference in the quantum yields.

Bleaching of ${ }^{3} \mathbf{N D S}^{2-}$ and $\mathbf{N D S}^{\mathbf{*}-}$ is accompanied by electron formation; in both cases, the amount of $\mathbf{e}_{\mathbf{a q}}{ }^{--}$produced equalled the amount of the precursor destroyed within $1 \%$. The intensity dependences are displayed in Fig. $5 \mathrm{~b}$. In the experiments on the radical anion, a small correction $(\leq 5 \%)$ had to be applied to take into account the triplet still present at the moment of the ionizing pulse (compare Fig. 3a). For the determination of the photoionization quantum yields, we again used relative actinometry with $\left[\mathrm{Ru}(\mathrm{bpy})_{3}\right]^{2+}$ as the standard. The MLCT state of $\left[\mathrm{Ru}(\mathrm{bpy})_{3}\right]^{2+}$ is itself photoionized at $355 \mathrm{~nm}$ but that process becomes only noticeable at higher pulse energies; ${ }^{34}$ by restricting the actinometry to the low-energy regime, we obtained photoionization quantum yields $\varphi_{355}$ at $355 \mathrm{~nm}$ of 0.08 for the triplet and 0.42 for the radical anion.

Whereas $\varphi_{355}$ for the triplet and $\varphi_{532}$ for the radical anion are the true quantum yields $\varphi_{1}$ for electron detachment from the lowest autoionizing state of the respective intermediate, $\varphi_{355}$ for the radical anion is a compound quantity comprising direct electron ejection from the upper state $D_{u}$ with the true quantum yield $\varphi_{\mathrm{u}}$ and indirect ejection from the lower state $\mathrm{D}_{\mathrm{l}}$ reached by radiationless deactivation (see the spectra in Section S2 of the ESI $\dagger$ ). That situation can be analyzed with the kinetic scheme given as the inset of Fig. 5b. Setting up the photokinetic equations in the usual way and rearranging leads to eqn (6) for $\varphi_{\mathrm{u}}$,

$$
\varphi_{\mathrm{u}}=\frac{\varphi_{355}-\varphi_{532}}{1-\varphi_{532}}
$$

Inserting the experimental quantities gives 0.20 for $\varphi_{\mathrm{u}}$. In view of the necessary approximations involved, in particular the neglect of individual rate constants for the vibrationally excited states of $D_{u}$ and $D_{1}$, the absolute number is not very meaningful but we take this result to indicate that direct electron ejection is slightly more likely for the lower state than for the higher despite the considerable energy difference.

The electronic configuration of the state reached by the second photon might provide an explanation. The following reasoning is based on a Koopmans' picture of photoionization, an approximation that has proved very successful for interpreting the photoelectron spectra of organic hydrocarbons with conjugated $\pi$-systems. ${ }^{36}$ Only in the case of a type-I excitation, meaning the promotion of an electron from the SOMO (singly occupied molecular orbital) to a virtual orbital, does the ionization of a radical anion recover the parent compound in its electronic ground state; type-II or type-III excitations, that is, from a doubly occupied orbital to the SOMO or to a virtual orbital, are correlated with an excited state of the substrate as the by-product of $\mathbf{e}_{\mathrm{aq}}{ }^{--} .^{-5}$ Because the singlet energy of $\mathbf{N D S}^{2-}$ is at least $3.8 \mathrm{eV}$, as calculated from the red edge of the absorption band (compare the spectrum in the ESI $\dagger$ ), type-II and type-III excitations should not lead to ionization of $\mathbf{N D S}^{\mathbf{0}^{3-}}$ at our laser wavelengths. By the same token, ionization of ${ }^{3} \mathbf{N D S}^{2-}$ is unlikely to be feasible by a transition other than from the higher SOMO to a virtual orbital.

The absorption spectra of the triplet and the radical anion of naphthalene have been thoroughly investigated and characterized. ${ }^{5,37-39}$ Because the substitution with the two sulfonate groups does not exert a significant influence on these spectra (compare, Sections S2.1-S2.3 of the ESI $\dagger$ ), we take the unsubstituted hydrocarbon as a model for $\mathbf{N D S}^{2-}$, and use the Mullikan symbols in $D_{2 \mathrm{~h}}$ for identification of the orbitals. Adopting the coordinate system of ref. 39, the lower SOMO of the triplet $\left(\mathrm{SOMO}_{\mathrm{l}}\right)$ is $1 \mathrm{a}_{\mathrm{u}}$, and the upper SOMO of the triplet $\left(\mathrm{SOMO}_{\mathrm{u}}\right)$ as well as the SOMO of the radical anion are $2 \mathrm{~b}_{1 \mathrm{~g}}$.

Owing to interactions with $\sigma^{*}$ orbitals, the transition SOMO $\rightarrow$ $\mathrm{SOMO}+2\left(2 \mathrm{~b}_{1 \mathrm{~g}} \rightarrow 3 \mathrm{~b}_{3 \mathrm{u}}\right)$ is responsible for both absorption bands of the radical anion in the visible; although the $\mathrm{B}_{3 \mathrm{~g}}$ states so reached lie above the ionization threshold, discrete absorption bands are still observed because of shape resonances. ${ }^{5}$ 
With $532 \mathrm{~nm}$, which corresponds to the minimum between the bands, both transitions are excited, but because they are both type-I transitions it should not make much of a difference whether the vibrational ground state of the higher-energy electronic state or a vibrationally excited state of the lower-energy electronic state is reached. The situation is different for $355 \mathrm{~nm}$ excitation. Although that wavelength practically coincides with the maximum of the near-UV band, this simplicity is deceptive: the band is a superposition of the type-II transition SOMO - $1 \rightarrow$ SOMO $\left(1 \mathrm{a}_{\mathrm{u}} \rightarrow 2 \mathrm{~b}_{1 \mathrm{~g}}\right)$ in addition to two-with the splitting again due to interactions with $\sigma^{*}$ orbitals - type-I transitions from the SOMO to the SOMO $+3\left(2 \mathrm{~b}_{1 \mathrm{~g}} \rightarrow 2 \mathrm{a}_{\mathrm{u}}\right){ }^{5}$. The fact that the absorption at $355 \mathrm{~nm}$ partly involves a nonionizing transition is consistent with the slightly smaller true quantum yield for ionization of NDS $^{\bullet 3-}$ with $355 \mathrm{~nm}$ compared to $532 \mathrm{~nm}$.

The type-I transition $\mathrm{SOMO}_{\mathrm{u}} \rightarrow \mathrm{SOMO}_{\mathrm{u}}+2\left(2 \mathrm{~b}_{1 \mathrm{~g}} \rightarrow 3 \mathrm{~b}_{3 \mathrm{u}}\right)$ contributes to the visible absorption band of the triplet but there is a dominant admixture of type-II transitions, the most important being $\mathrm{SOMO}_{1}-3 \rightarrow \mathrm{SOMO}_{\mathrm{u}}+2$, i.e., $1 \mathrm{~b}_{1 \mathrm{~g}} \rightarrow 3 \mathrm{~b}_{3 \mathrm{u} \cdot}{ }^{38}$ The next band at higher energy is too far in the UV-C for its wings to be noticeably excited with $355 \mathrm{~nm}$ and furthermore contains only $50 \%$ of a type-I transition. ${ }^{37}$ The low quantum yield of $355 \mathrm{~nm}$ photoionization of ${ }^{3} \mathbf{N D S}^{2-}$ can thus be rationalized with the same arguments as above, in addition to the shorter life of the higher excited state.

\section{Experimental section}

Our investigation method was two-color laser flash photolysis with optical detection of the intermediates. The setup is described in detail in Section $\mathrm{S} 1$ of the ESI. $\dagger$

The chemicals were obtained commercially in the highest available purity and used as received (disodium 1,5-naphthalene disulfonate hydrate, $>98 \%$, TCI; sodium ascorbate, $>99 \%$, Alfa Aesar; ruthenium(tris)bipyridine dichloride hexahydrate, 99\%, abcr; sodium chloride, $>99.5 \%$, Fluka). Ultrapure Millipor MilliQ water (specific resistance, $18.2 \mathrm{M} \Omega \mathrm{cm}$ ) was the solvent. All solutions were purged with argon (5.0, Air Liquide) or $\mathrm{N}_{2} \mathrm{O}$ (4.5, Linde) for 30 minutes before and for the whole duration of the experiments. To avoid the well-known oxidation of sodium ascorbate, ${ }^{40}$ we added the required amount in solid form to the already degassed solutions immediately before starting the experiments. We employed the substrate $\mathbf{N D S}^{\mathbf{2}-}$ as its disodium salt $\mathbf{N a}_{2} \mathbf{N D S}$ because that does not affect the natural $\mathrm{pH}$ of our aqueous solutions, in contrast to the disulfonic acid. As $\mathbf{N a}_{2} \mathbf{N D S}$ is hygroscopic, we determined its water content $(7.02 \%)$ by a thermogravimetric analysis (TA Instruments TGA2950) to obtain the exact substrate concentration.

All steady-state absorption spectra were recorded with a Shimadzu UV-2102 spectrophotometer.

\section{Conclusions}

Of the very few known monophotonic green-light ionizations in polar solvents, ${ }^{35,41-44}$ that of the doubly deprotonated terephthalate radical anion has the highest quantum yield $\left(\varphi_{\text {ion }}=0.07\right)^{35}$ reported so far. The system studied in the present work constitutes a very significant advance because $\varphi_{\text {ion }}$ is four times as high for it, which compares favourably with the quantum yields of the most efficient UV photoionizations known to date, ${ }^{6}$ although the energy of the ionizing photon is lower by almost $200 \mathrm{~kJ} \mathrm{~mol}^{-1}$. As our investigation of the wavelength dependence has shown, however, the energy balance only provides a criterion of whether a photoionization is basically feasible or not but has little predictive power for the quantum yield; our results suggest that the electronic configuration of the excited electron precursor is a far more important factor.

Similar substrates with more highly conjugated aromatic systems appear very promising candidates for optimizing the cyclic photoionization mechanism of Fig. 1b. First, the photochemical generation of their radical anions is possible with light of longer wavelengths, even in the visible range, thus making more effective use of the solar spectrum. Second, aryl radical anions in general are sufficiently energy-rich ${ }^{45}$ for their green-light photoionization to be feasible, but also comparatively long-lived, ${ }^{27,46}$ thus increasing the probability of absorbing the ionizing photon at much lower light levels than those of a pulsed laser.

\section{Acknowledgements}

Financial support provided by the Chemical Industry Funds to C.K. is gratefully acknowledged. We thank Till Walther for performing the TGA measurement.

\section{References}

1 J. W. T. Spinks and R. J. Woods, An Introduction to Radiation Chemistry, Wiley, New York, 2nd edn, 1976.

2 X. Li, J. Ma, G. Liu, J. Fang, S. Yue, Y. Guan, L. Chen and X. Liu, Environ. Sci. Technol., 2012, 46, 7342-7349.

3 Y. Peng, S. He, J. Wang and W. Gong, Radiat. Phys. Chem., 2012, 81, 1629-1633.

4 Z. Song, H. Tang, N. Wang and L. Zhu, J. Hazard. Mater., 2013, 262, 332-338.

5 Y. Honda, T. Shida and H. Nakatsuji, J. Phys. Chem. A, 2012, 116, 11833-11845.

6 F. Saito, S. Tobita and H. Shizuka, J. Photochem. Photobiol., A, 1997, 106, 119-126.

7 M. Wang, L.-L. Cheng, H. Zhu, K. Li, Q.-S. Wu, S.-D. Yao and S.-L. Wang, J. Photochem. Photobiol., A, 2009, 208, 104-109.

8 M. P. Yurkova, I. P. Pozdnyakov, V. F. Plyusnin, V. P. Grivin, N. M. Bazhin, A. I. Kruppa and T. A. Maksimova, Photochem. Photobiol. Sci., 2013, 12, 684-689.

9 L.-L. Cheng, M. Wang, H. Zhu, K. Li, R.-R. Zhu, X.-Y. Sun, S.-D. Yao, Q.-S. Wu and S.-L. Wang, Spectrochim. Acta, Part A, 2009, 73, 955-959.

10 M. Goez and B. H. M. Hussein, Phys. Chem. Chem. Phys., 2004, 6, 5490-5497.

11 M. Goez and V. Zubarev, Chem. Phys., 2004, 307, 15-26. 
12 M. Goez and V. Zubarev, J. Phys. Chem. A, 1999, 103, 9605-9613.

13 M. Goez, V. Zubarev and G. Eckert, J. Am. Chem. Soc., 1998, 120, 5347-5348.

14 M. Goez and V. Zubarev, Angew. Chem., Int. Ed., 2006, 45, 2135-2138.

15 http://rredc.nrel.gov/solar/spectra/am1.5/ASTMG173/ASTMG173. html, retrieved 15.09.2014.

16 G. J. Kavarnos and N. J. Turro, Chem. Rev., 1986, 86, 401-449.

17 M. Nemoto, H. Kokubun and M. Koizumi, Bull. Chem. Soc. Jpn., 1969, 42, 2464-2470.

18 M. Goez and C. Kerzig, Angew. Chem., Int. Ed., 2012, 51, 12606-12608.

19 S. Steenken, C. J. Warren and B. C. Gilbert, J. Chem. Soc., Perkin Trans. 2, 1990, 335-342.

20 S. E. Braslavsky, Pure Appl. Chem., 2007, 79, 293-465.

21 P. Wardman, J. Phys. Chem. Ref. Data, 1989, 18, 1637-1755.

22 A. P. Shestov and N. A. Osipova, Sb. Statei, Nauchno-Issled. Inst. Org. Poluprod. Krasitelei, 1961, 2, 13-45.

23 K. Kasama, A. Takematsu and S. Arai, J. Phys. Chem., 1982, 86, 2420-2427.

24 A. A. Frost and R. G. Pearson, Kinetics and Mechanism, John Wiley and Sons, New York, 2nd edn, 1961.

25 M. Goez and I. Sartorius, J. Phys. Chem. A, 2003, 107, 8539-8546.

26 J. H. Fendler, H. A. Gillis and N. V. Klassen, J. Chem. Soc., Faraday Trans. 1, 1974, 70, 145-153.

27 A. Treinin and E. Hayon, J. Am. Chem. Soc., 1976, 98, 3884-3891.

28 R. Kaptein and L. J. Oosterhoff, Chem. Phys. Lett., 1969, 4, 195-197.

29 V. D. Parker, M. Tilset and O. Hammerich, J. Am. Chem. Soc., 1987, 7905-7906.
30 Z. V. Todres, Ion-Radical Organic Chemistry: Principles and Applications, CRC Press, Boca Raton, 2nd edn, 2008.

31 U. Lachish, A. Shafferman and G. Stein, J. Chem. Phys., 1976, 64, 4205-4211.

32 S. M. Hubig, J. Lumin., 1991, 47, 137-145.

33 S. Campagna, F. Puntoriero, F. Nastasi, G. Bergamini and V. Balzani, Top. Curr. Chem., 2007, 280, 117-214.

34 M. Goez, D. von Ramin-Marro, M. H. O. Musa and M. Schiewek, J. Phys. Chem. A, 2004, 108, 1090-1100.

35 P. Natarajan and R. W. Fessenden, J. Phys. Chem., 1989, 93, 6095-6100.

36 S. F. Nelsen, M. N. Weaver, D. Yamazaki, K. Komatsu, R. Rathore and T. Bally, J. Phys. Chem. A, 2007, 111, 1667-1676.

37 T.-K. Ha and U. P. Wild, J. Comput. Chem., 1982, 3, 1-5.

38 H. Chakraborty and A. Shukla, ArXiv e-prints, 2014, arXiv:1406.6954.

39 T. Bally, C. Carra, M. P. Fülscher and Z. Zhu, J. Chem. Soc., Perkin Trans. 2, 1998, 1759-1765.

40 M. B. Davies, J. Austin and D. A. Partridge, Vitamin C: Its Chemistry and Biochemistry, The Royal Society of Chemistry, Cambridge, 1st edn, 1991.

41 M. Hara, S. Samori, X. Cai, M. Fujitsuka and T. Majima, J. Phys. Chem. A, 2005, 109, 9831-9835.

42 J. K. Thomas and P. L. Piciulo, J. Am. Chem. Soc., 1979, 101, 2502-2503.

43 L. J. Johnston and R. W. Redmond, J. Phys. Chem. A, 1997, 101, 4660-4665.

44 R. W. Redmond, J. C. Scaiano and L. J. Johnston, J. Am. Chem. Soc., 1990, 112, 398-402.

45 M. Montalti, A. Credi, L. Prodi and M. T. Gandolfi, Handbook of Photochemistry, Taylor and Francis, Boca Raton, 3rd edn, 2006.

46 Y. Yamamoto, S. Shiraki and Y. Kawamura, J. Chem. Soc., Perkin Trans. 2, 1992, 2241-2245. 